\title{
Efeito da adição de polímero precursor cerâmico na sinterização de alumina
}

\author{
(Effect of the addition of ceramic polymeric \\ precursor on alumina sintering)
}

\author{
A.L.E.Godoy, J.C.Bressiani,A.H.A.Bressiani \\ CCTM - Instituto de Pesquisas Energéticas e Nucleares, Av. Prof. Lineu Prestes 2242, S. Paulo, SP 05508-000 \\ analucia@ipen.br
}

\begin{abstract}
Resumo
Foram estudados os efeitos da adição de polímero precursor na sinterização, microestrutura e dureza da alumina. O polímero inorgânico polifenilmetilvinilhidrogenosilsesquioxano (PPS) foi utilizado como aditivo em cerâmicas de alumina sinterizadas a $1650{ }^{\circ} \mathrm{C}$. Os materiais sinterizados foram caracterizados por dilatometria, determinando-se a densidade aparente por picnometria de hélio, pela análise de carbono total, por difração de raios X, por microscopia eletrônica de varredura, espectrometria de energia dispersiva de raios $\mathrm{X}$ e por análise de indentação Vickers para determinação de dureza e tenacidade a fratura. Foram obtidas fases heterogêneas de $\mathrm{Si}_{2} \mathrm{Al}_{4} \mathrm{O}_{4} \mathrm{~N}_{4}$ nas amostras com PPS. Dentre as amostras analisadas, a que contém 5\% de PPS apresentou dureza e tenacidade à fratura superiores à da alumina pura. A preparação de compósitos cerâmicos com pequenas quantidades de polímero precursor mostrou-se um processo adequado para cerâmicas à base de alumina, sendo uma rota simples de conformação com grande potencial para a obtenção de peças com geometria complexa.
\end{abstract}

Palavras-chave: polímero inorgânico, polissiloxano, microestrutura, alumina.

\begin{abstract}
The effects of the addition of precursor polymer on sintering, microstructure and hardness of alumina ceramics were studied. The linear shrinkage of cold-pressed specimens was studied by dilatometry. Polyphenylmethylvinylhidrogensilsesquioxane (PPS) polymer sintering aid was used for alumina based ceramics sintered at $1650{ }^{\circ} \mathrm{C}$. The sintered materials were characterized by determination of apparent density by helium picnometry, by evaluation of total carbon content, X-ray diffraction, scanning electron microscopy, energy dispersive X-ray spectrometry, and by Vickers indentation analysis for determining hardness and fracture toughness. Heterogeneous $\mathrm{Si}_{2} \mathrm{Al}_{4} \mathrm{O}_{4}$ phases were obtained in specimens with PPS. The specimens with $5 \%$ of PPS presented hardness and toughness greater than the obtained for pure alumina. The preparation of ceramic composites using small amounts of precursor polymers shows a suitable process for alumina-based ceramics, a simple forming route, with high potential for the fabrication of complex shape pieces.
\end{abstract}

Keywords: inorganic polymer, polysiloxane, microstructure, alumina.

\section{INTRODUÇÃO}

As cerâmicas estruturais, tais como alumina, nitreto de silício, carbeto de silício e zircônia possuem várias vantagens em relação ao aço, como estabilidade química e térmica a elevadas temperaturas e excelente resistência ao desgaste. Recentemente houve um aumento na utilização de alumina de alta pureza devido à sua aplicação em semicondutores e painéis de cristal líquido [1]. Nas últimas décadas [2,3] têm sido desenvolvidos novos materiais, por exemplo, compósitos, que têm sido muito estudados com o intuito de melhorar algumas propriedades dos materiais cerâmicos, como a baixa tenacidade à fratura.

O desenvolvimento de novos polímeros inorgânicos ou organo-metálicos tem permitido a obtenção de novos materiais para utilização em diversas áreas, principalmente em altas temperaturas e ambientes quimicamente agressivos $[4,5]$. Existem diversos trabalhos envolvendo a utilização de polímeros precursores cerâmicos [6,7]. A síntese de cerâmica a partir de polímeros precursores, como fibras, filmes finos, espumas, ligantes, nanofibras, recobrimentos, vidros e materiais compactados têm sido alvo de atenção especial por parte de pesquisadores [8-15]. Nos últimos anos foram desenvolvidos diversos polímeros inorgânicos, sendo que o comportamento químico dos mesmos depende da natureza dos grupos funcionais $\mathrm{R}$ ligados aos átomos de $\mathrm{Si}$, como grupos metílicos e fenílicos. Os polissiloxanos são polímeros obtidos por reações de hidrólise/condensação de misturas de clorossilanos [5, 16]. Dentre os polímeros precursores cerâmicos, os polissiloxanos são materiais 
relativamente baratos, apresentam considerável estabilidade térmica, possuem excelentes propriedades elétricas e hidrofóbicas, baixa tensão superficial e baixa temperatura de transição vítrea. Comparados aos polímeros livres de oxigênio, os polissiloxanos são mais fáceis de manusear, de serem processados em condições ambientais, apresentando numerosas aplicações industriais, como isolantes elétricos, agentes anti-espumantes, membranas, adesivos, etc. [8]. Os polissiloxanos, além de serem estáveis em atmosfera oxidante, possuem métodos químicos para a reticulação bem estabelecidos [9], sendo capazes de gerar estruturas bastante reticuladas, minimizando a eliminação de moléculas de baixa massa molar, freqüentemente responsáveis pela perda de massa a temperaturas relativamente baixas [10]. A ligação entre os átomos de $\mathrm{Si}$ e $\mathrm{O}$ na silicona é forte, porém flexível, de forma que este material possui alta resistência térmica, sem se decompor. A flexibilidade é proveniente da movimentação do ângulo formado pelas ligações entre os átomos de Si e O, Fig. 1 [17]. Dependendo do comprimento da cadeia e dos grupos orgânicos ligados aos átomos de silício pode-se obter desde óleos pouco viscosos até graxas, géis, borrachas, resinas sólidas, selantes, óleos lubrificantes, fluidos para uso em hidráulica, biomateriais e cosméticos, entre outros [18]. Siliconas, que não são derivadas do petróleo, têm sido comercializadas desde os anos 1940 tendo, desde então, várias aplicações industriais [18]. Podem também ser o insumo para obtenção de materiais cerâmicos do tipo $\mathrm{Si} / \mathrm{C} / \mathrm{O}$, chamados oxicarbetos de silício, oferecendo uma rota fácil e de custo relativamente baixo $[19,20]$ na formação deste sistema. A conversão polímero-cerâmica geralmente é acompanhada por retração e volatilização de orgânicos, causando perda de massa [21].

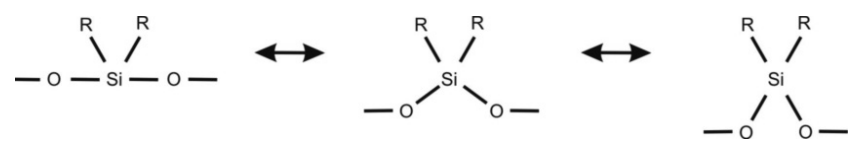

Figura 1: Esquema representando o movimento da cadeia principal de silicona, conferindo flexibilidade ao material [17].

[Figure 1: Schematic drawing of motion of the main chain of silicon, giving materials flexibility [17].]

$\mathrm{O}$ compósito obtido neste trabalho é à base de $\mathrm{Al}_{2} \mathrm{O}_{3}$, material muito utilizado devido à excelente combinação de propriedades físicas e químicas, tais como, boas resistências ao desgaste e à oxidação e estabilidades térmica e química [22-24]. A alumina é utilizada como revestimentos cerâmicos, abrasivos, mobília para fornos, ferramentas de corte, componentes para uso médico e odontológico [24, 25], entre outras.

O processamento de compósitos cerâmicos com a utilização de polímeros precursores possui uma vasta gama de possibilidades, como o processo AFCOP (Active Filler Controlled Polymer Pyrolysis - Pirólise Controlada de Polímeros e Carga Ativa) [5], permitindo obter peças com formas complexas como discos, parafusos, etc. [26].

O objetivo deste trabalho é o estudo do efeito de adição de até $10 \%$ em massa do polímero precursor cerâmico, polissiloxano, na sinterização, microestrutura e dureza da alumina.

\section{MATERIAIS E MÉTODOS}

As matérias-primas utilizadas foram óxido de alumínio, $\mathrm{Al}_{2} \mathrm{O}_{3}$ A16SG (Alcoa), polifenilmetilvinilhidrogenosilsesquioxano (PPS) - H62C, líquido de baixa viscosidade na temperatura ambiente, $1,4 \cdot 10^{-3} \mathrm{~m}^{2} \cdot \mathrm{s}^{-1}$, composição molecular aproximada $\left(\mathrm{RSiO}_{1,5}\right)_{\mathrm{n}}$, na qual $\mathrm{R}$ é $\left[\left(\mathrm{C}_{6} \mathrm{H}_{5}\right)_{2,8},\left(\mathrm{CH}_{3}\right)_{1,5}\right.$, $\left.\left(\mathrm{CH}_{2}=\mathrm{CH}\right),(\mathrm{H})\right]$ - MK- Wacker Chemie e catalisador com platina - Hüll. Foram feitas adições de $1 \%, 5 \%$ e $10 \%$ em massa de PPS em $\mathrm{Al}_{2} \mathrm{O}_{3}$. O processamento das amostras incluiu moagem de $\mathrm{Al}_{2} \mathrm{O}_{3}$ em moinho atritor, com álcool isopropílico, haste, jarro e esferas de alumina, por $4 \mathrm{~h}$. O material foi seco em rotoevaporador, desaglomerado em almofariz de ágata e passado em peneira (100 mesh). A amostra padrão, sem adição de polímero, denominada A0, foi compactada uniaxialmente e em prensa isostática a $200 \mathrm{MPa}$ e sinterizada a $1650{ }^{\circ} \mathrm{C}$, em forno de resistência de grafite (Astro) e pressão positiva de nitrogênio. Nas outras amostras o catalisador com Pt, diluído em álcool isopropílico, foi adicionado à mistura de polímero e $\mathrm{Al}_{2} \mathrm{O}_{3}$. A homogeneização do material foi feita em misturador mecânico (Túrbula T2C), o material foi seco a $50{ }^{\circ} \mathrm{C}$ por 30 min e compactado uniaxialmente em prensa a quente a $200{ }^{\circ} \mathrm{C}$ por $2 \mathrm{~h}$. As amostras foram submetidas a processo de pirólise a $900{ }^{\circ} \mathrm{C}$ com patamar de $1 \mathrm{~h}$ em forno tubular sob atmosfera dinâmica de nitrogênio.A sinterização foi realizada em forno de resistência de grafite a $1650{ }^{\circ} \mathrm{C}$ com patamar de $1 \mathrm{~h}$ e pressão positiva de nitrogênio. Este tratamento térmico foi feito em cadinho fechado e cama protetora de alumina. A taxa de aquecimento foi $5{ }^{\circ} \mathrm{C} / \mathrm{min}$ até $400{ }^{\circ} \mathrm{C}$ e $20^{\circ} \mathrm{C} / \mathrm{min}$ de $400{ }^{\circ} \mathrm{C}$ até a temperatura de sinterização.

A densidade aparente das amostras sinterizadas foi obtida por picnometria de $\mathrm{He}$ (Micromeritics 1330). Foram determinadas também as perdas de massa. A distribuição granulométrica do pó de $\mathrm{Al}_{2} \mathrm{O}_{3}$ foi determinada por difração de laser (Cilas 1064), utilizando como agente dispersante duramax. Foi feita análise termogravimétrica do polímero PPS para determinação da variação de massa em função da temperatura em termobalança Shimadzu TGA $50 \mathrm{H}$. As amostras foram aquecidas até $1350{ }^{\circ} \mathrm{C}$ com taxa de aquecimento $10{ }^{\circ} \mathrm{C} / \mathrm{min}$ sob fluxo $(10 \mathrm{~mL} / \mathrm{min})$ de nitrogênio de alta pureza. A retração linear do material compactado com $10 \%$ em massa de polímero foi obtida por dilatometria (Netzsch DIL/C7) até $1650{ }^{\circ} \mathrm{C}$, sob nitrogênio. A taxa de aquecimento foi $10^{\circ} \mathrm{C} / \mathrm{min}$ até $1650^{\circ} \mathrm{C}$, patamar de $1 \mathrm{~h} \mathrm{e}$ resfriamento de $10^{\circ} \mathrm{C} / \mathrm{min}$. Para a análise das fases cristalinas nas amostras sinterizadas foi utilizado difratômetro de raios X (Rigaku Multiflex), com radiação $\mathrm{Cuk}_{\alpha}$. As amostras foram observadas em microscópio eletrônico de varredura (Philips XL30) e analisadas em espectrômetro de dispersão de energia de raios X (EDS, Philips). Foram feitos ensaios de impressão Vickers em máquina de dureza Buehler VMT7, 10 impressões em cada amostra, levando-se em conta o distanciamento adequado sugerido [27]. Para a análise do 
tipo das trincas decorrentes das impressões Vickers foi feito polimento das amostras em pasta de diamante $6 \mu \mathrm{m}$ por 2 min. A equação utilizada para o cálculo da tenacidade à fratura para trinca radial-mediana é [28]:

$$
\mathrm{K}_{\mathrm{Ic}}=0,0154\left(\mathrm{E} / \mathrm{H}_{\mathrm{v}}\right)^{1 / 2}\left(\mathrm{P} / \mathrm{c}^{3 / 2}\right)
$$

sendo $\mathrm{K}_{\mathrm{Ic}}$ a tenacidade à fratura $\left(\mathrm{MPa} \cdot \mathrm{m}^{1 / 2}\right)$, E o módulo de elasticidade (GPa), $\mathrm{H}_{\mathrm{v}}$ a dureza Vickers (MPa), P a carga aplicada $(\mathrm{N})$ e c o comprimento da trinca $(\mu \mathrm{m})$. Foi utilizado analisador de carbono total via absorção de radiação infravermelho acoplado a um forno de radiofreqüência (Leco CS400), para análise da quantidade de carbono das amostras de $\mathrm{Al}_{2} \mathrm{O}_{3}$ :PPS após sinterização.

As amostras foram codificadas: A0 é a amostra sem adição de polímeros e $\mathbf{A X}$ representam as amostras de alumina com adição ( $\mathbf{X}=1,5$ ou $10 \%$ em massa) do polímero PPS.

\section{RESULTADOS E DISCUSSÃO}

Nas Figs. 2a e 2b são mostradas as micrografias dos pós de $\mathrm{Al}_{2} \mathrm{O}_{3}$ e da mistura de $\mathrm{Al}_{2} \mathrm{O}_{3}$ com $10 \%$ de PPS, após secagem. De acordo com resultados obtidos por difração de laser, o diâmetro médio das partículas do pó de $\mathrm{Al}_{2} \mathrm{O}_{3}$ é $0,64 \mu \mathrm{m}$, elas são arredondadas e não estão aglomeradas. Estes pós foram observados como recebidos. $\mathrm{Na}$ análise morfológica de $\mathrm{Al}_{2} \mathrm{O}_{3}: 10 \%$ PPS observa-se características semelhantes às partículas do pó como recebido, Fig. 2a, a não ser pelo fato que, com a adição de polímero, as mesmas encontram-se um pouco aglomeradas.

Como a atmosfera interfere na degradação dos polímeros [29], foi feito ensaio por termogravimetria em atmosfera de nitrogênio, Fig. 3. O início do processo de decomposição térmica de polissiloxanos é devido, principalmente, a reações de degradação que ocorrem por meio de rearranjos inter- e intracadeias, gerando estruturas voláteis [30]. O polímero PPS, sob atmosfera de nitrogênio, entre $180{ }^{\circ} \mathrm{C}$ e $400{ }^{\circ} \mathrm{C}$ apresenta pequena perda de massa, porém entre $400{ }^{\circ} \mathrm{C}$ e $800{ }^{\circ} \mathrm{C}$ há perda de massa acentuada. Neste intervalo de temperatura são quebradas as ligações $\mathrm{Si}^{-} \mathrm{C}_{6} \mathrm{H}_{5}$ com liberação de $\mathrm{C}_{6} \mathrm{H}_{6}$ [31]. Há também a liberação de $\mathrm{CH}_{4}$ e $\mathrm{H}_{2}$, com a "ceramização" do material. "Ceramização" na pirólise corresponde à fase do processo na qual ocorre a conversão do material de estrutura polimérica para estrutura cerâmica amorfa ou cristalina [32]. A massa remanescente a $1350{ }^{\circ} \mathrm{C}$ foi de aproximadamente $73 \%$.

Os polissiloxanos, em geral, são estáveis em vácuo ou em atmosfera inerte até cerca de $350{ }^{\circ} \mathrm{C}$ a $400{ }^{\circ} \mathrm{C}$ [33]. A alta estabilidade térmica dos polissiloxanos é atribuída à alta energia e caráter iônico das ligações Si-O $(443,7$ kJ/mol) em relação à ligação de C-C $(345,7 \mathrm{~kJ} / \mathrm{mol})$ [33]. A decomposição térmica dos polissiloxanos ocorre principalmente devido à quebra de ligações do $\mathrm{Si}-\mathrm{O}$, que constituem sua cadeia principal [34].

A Fig. 4 mostra a curva de retração linear em função da temperatura para a amostra A10. Nota-se um pequeno declive em torno de $800{ }^{\circ} \mathrm{C}$, que pode ser devido à volatilização
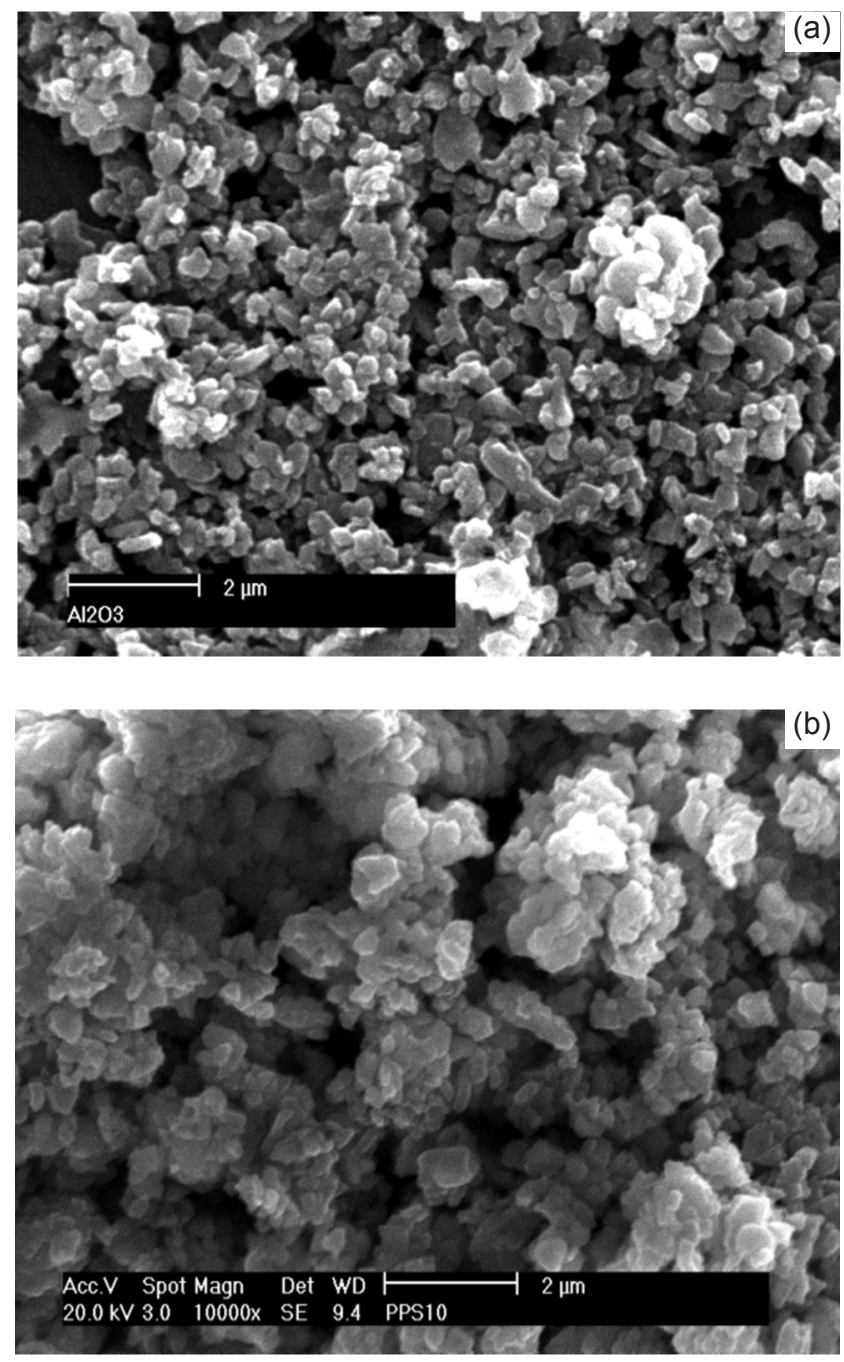

Figura 2: Micrografias de pós utilizados como matéria-prima, obtidas por MEV: (a) $\mathrm{Al}_{2} \mathrm{O}_{3}$; (b) mistura $\mathrm{Al}_{2} \mathrm{O}_{3}: 10 \%$ PPS.

[Figure 2: SEM micrographs of the powders: (a) $\mathrm{Al}_{2} \mathrm{O}_{3}$; (b) $\mathrm{Al}_{2} \mathrm{O}_{3}$ : PPS mixture.]

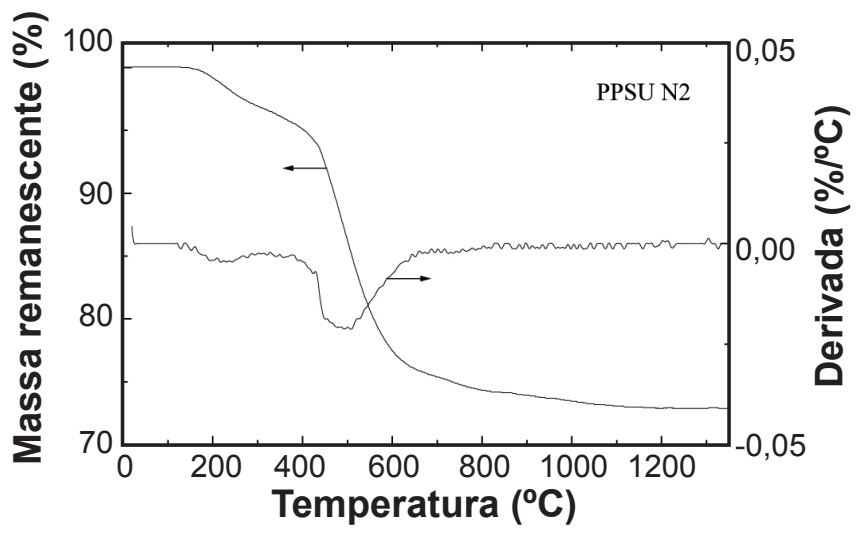

Figura 3: Curvas TG/DTG do polímero PPS. [Figure 3: TG/DTG curves of PPS polymer.]

do polímero. Em aproximadamente $1200{ }^{\circ} \mathrm{C}$ a retração é mais acentuada, com uma inflexão em cerca de $1550{ }^{\circ} \mathrm{C}$, que significa que possivelmente houve formação de fase $\mathrm{e}$ 


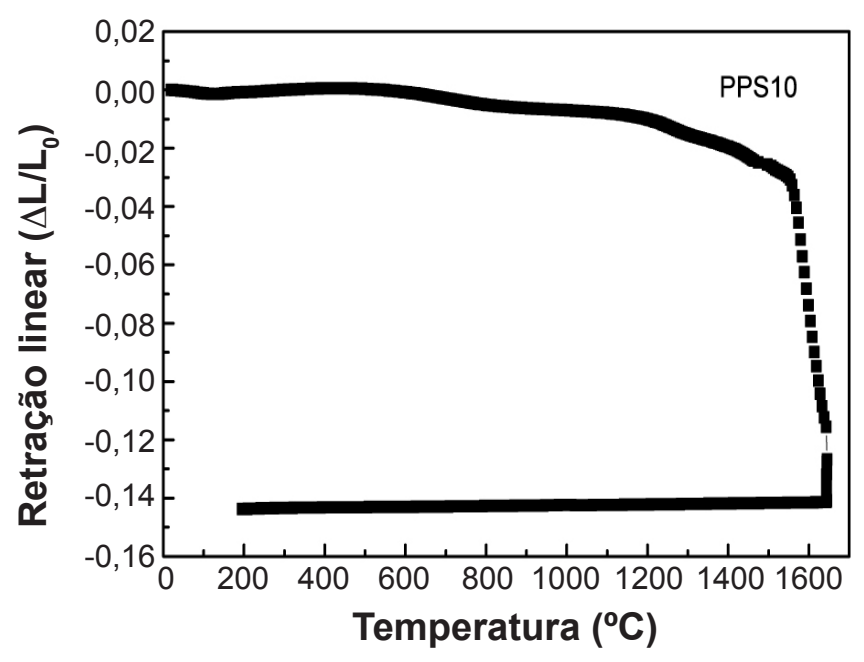

Figura 4: Retração linear da amostra A10: 1- aquecimento; 2patamar de $1 \mathrm{~h}$ a $1650^{\circ} \mathrm{C}$ e 3- resfriamento.

[Figure 4: A10 linear shrinkage curve: 1- heating; 2- holding time of 1 h at $1650^{\circ} \mathrm{C}$ and 3 - cooling.]

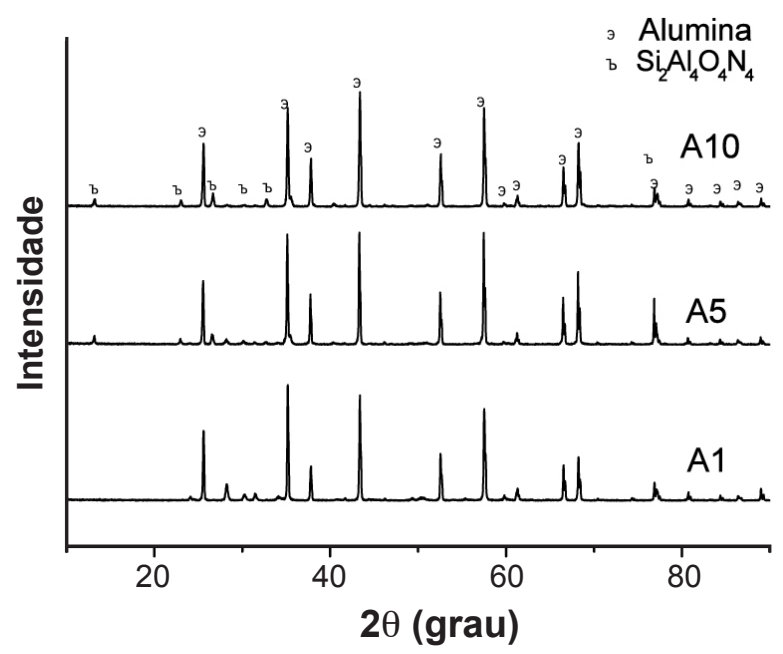

Figura 5: Difratogramas de raios $X$ das amostras A1, A5 e A10 após pirólise e sinterização em atmosfera de nitrogênio.

[Figure 5: X-ray diffraction patterns of A1, A5 and A10 specimens after pyrolysis and sintering under nitrogen.]

Tabela I - Densidade aparente, \% D.T. e perda de massa das amostras A0, A1, A5 e A10.

[Table I - Apparent density, \%TD and loss of mass during pyrolysis and sintering of A0, A1, A5 and A10 specimens.]

\begin{tabular}{cccccc}
\hline Amostra & $\begin{array}{c}\rho_{\text {aparente }} \\
\left(\mathrm{g} / \mathrm{cm}^{3}\right)\end{array}$ & $\begin{array}{c}\text { D.T. } \\
\%\end{array}$ & $\begin{array}{c}\mathrm{PM} \\
\mathrm{B} \rightarrow \mathrm{A} \\
(\%)\end{array}$ & $\begin{array}{c}\mathrm{PM} \\
\mathrm{C} \rightarrow \mathrm{B} \\
(\%)\end{array}$ & $\begin{array}{c}\mathrm{PM} \\
\mathrm{C} \rightarrow \mathrm{A} \\
(\%)\end{array}$ \\
\hline A0 & $3,96 \pm 0,03$ & 99,5 & --- & --- & 1,2 \\
A1 & $3,84 \pm 0,04$ & 96,5 & 0,1 & 1,0 & 1,1 \\
A5 & $3,71 \pm 0,05$ & 93,2 & 1,3 & 1,6 & 2,9 \\
A10 & $3,78 \pm 0,04$ & 95,0 & 2,3 & 3,9 & 6,1 \\
\hline
\end{tabular}

$\mathbf{A} \rightarrow$ antes da pirólise $\quad \mathbf{B} \rightarrow$ após pirólise $\mathbf{C} \rightarrow$ após sinterização

$\mathbf{B} \rightarrow \mathbf{A}$ - perda de massa após pirólise

$\mathbf{C} \rightarrow \mathbf{B}$ - perda de massa parcial entre pirólise e sinterização

$\mathbf{C} \rightarrow \mathbf{A}$ - perda de massa total

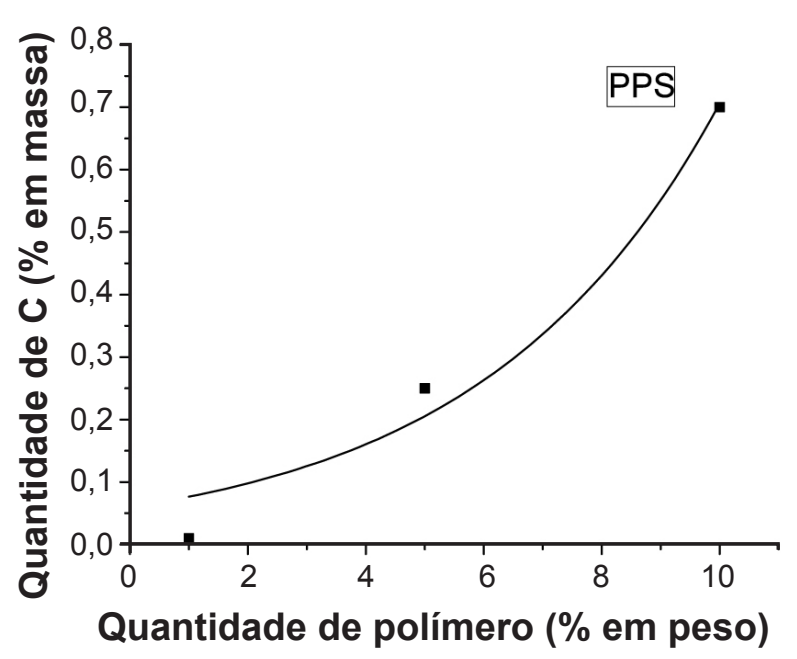

Figura 6: Análise de carbono total após sinterização das amostras A1, A5 e A10.

[Figure 6: Analysis of total carbon content of A1, A5 and A10 samples after sintering]. alterações nos mecanismos de sinterização. Na região de patamar $\left(1650^{\circ} \mathrm{C} / 1 \mathrm{~h}\right)$ o material ainda está retraindo. A retração máxima foi de aproximadamente $14 \%$.

Na Fig. 5 são mostrados os difratogramas de raios $X$ das amostras A1, A5 e A10. Foram identificadas as fases $\mathrm{Al}_{2} \mathrm{O}_{3}$ (JCPDS 26-31) e $\mathrm{Si}_{2} \mathrm{Al}_{4} \mathrm{O}_{4} \mathrm{~N}_{4}$ (JCPDS 76-598) nas amostras de $\mathrm{Al}_{2} \mathrm{O}_{3}:$ PPS. A presença de $\mathrm{Si}_{2} \mathrm{Al}_{4} \mathrm{O}_{4} \mathrm{~N}_{4}$ está relacionada às características físicas da $\mathrm{Al}_{2} \mathrm{O}_{3}$. Este pó é fino, portanto reativo, possibilitando a ocorrência de reações entre $\mathrm{Al}_{2} \mathrm{O}_{3}$ e os produtos de decomposição do polímero, como $\mathrm{Si}, \mathrm{O}$ e atmosfera de $\mathrm{N}_{2}$, gerando novas fases [32]. Por outro lado, $\mathrm{Si}_{2} \mathrm{Al}_{4} \mathrm{O}_{4} \mathrm{~N}_{4}$ é um material de relevante importância, já que possui boa resistência à flexão e alta resistência ao desgaste.

As densidades aparentes, \% D.T. e perdas de massa das amostras nas diversas etapas de processamento são apresentadas na Tabela I. A\% D.T. foi calculada considerando como densidade teórica a da alumina pura $\left(3,98 \mathrm{~g} / \mathrm{cm}^{3}\right.$, JCPDS 26-31). A alumina pura, material de referência, possui densidade superior às obtidas com amostras preparadas 


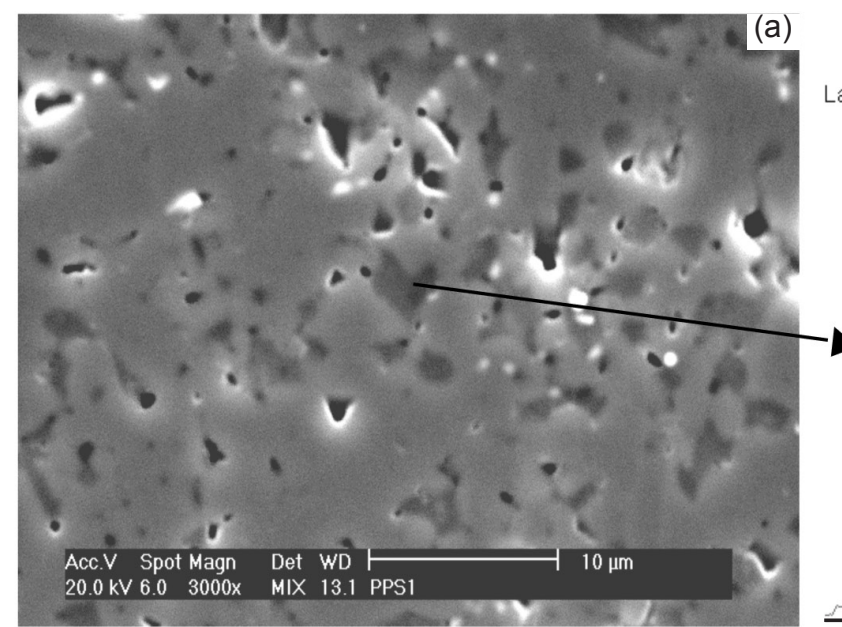

(c)

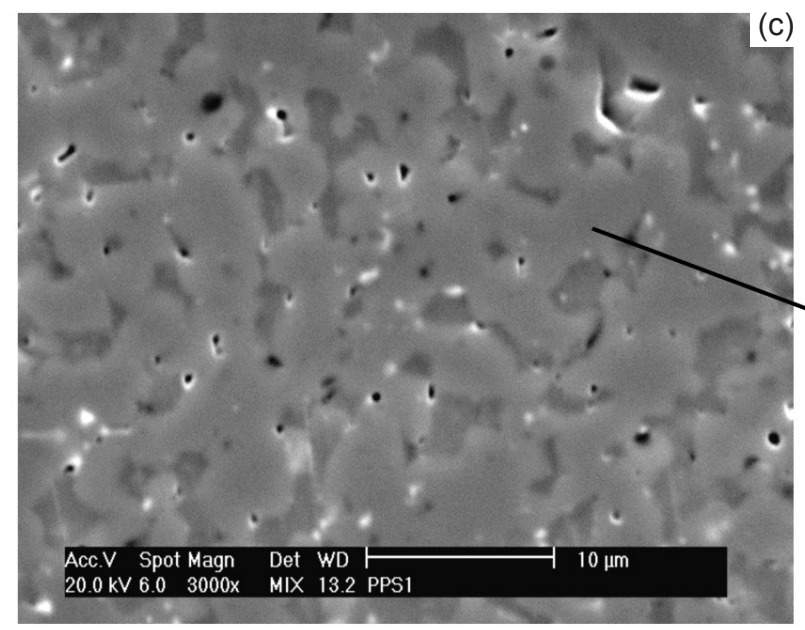

Energia (keV)

(b)

Label A: PPS1 FASE CINZA

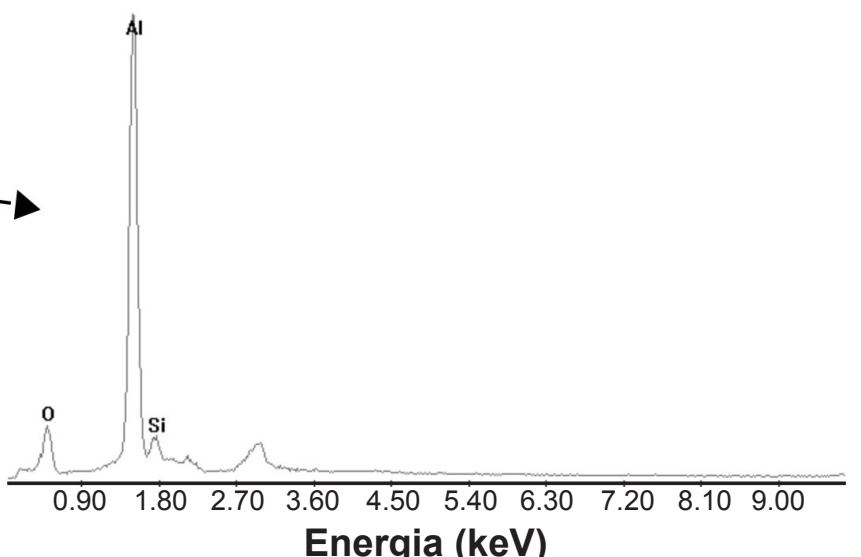

(d)

Label A: PPS1 MATRIZ

\section{Energia (keV)}

Figura 7: Micrografias obtidas por MEV da amostra A1P: (a) amostra polida, sem ataque; (b) EDS da região marcada com setas, apresentada no item (a); (c) Amostra polida, sem ataque; (d) EDS da matriz.

[Figure 7: SEM micrographs of AlP sample: (a) polished sample without etching; (b) EDS spectrum of region presented in item (a) polished sample without etching; (d) matrix EDS spectrum.]
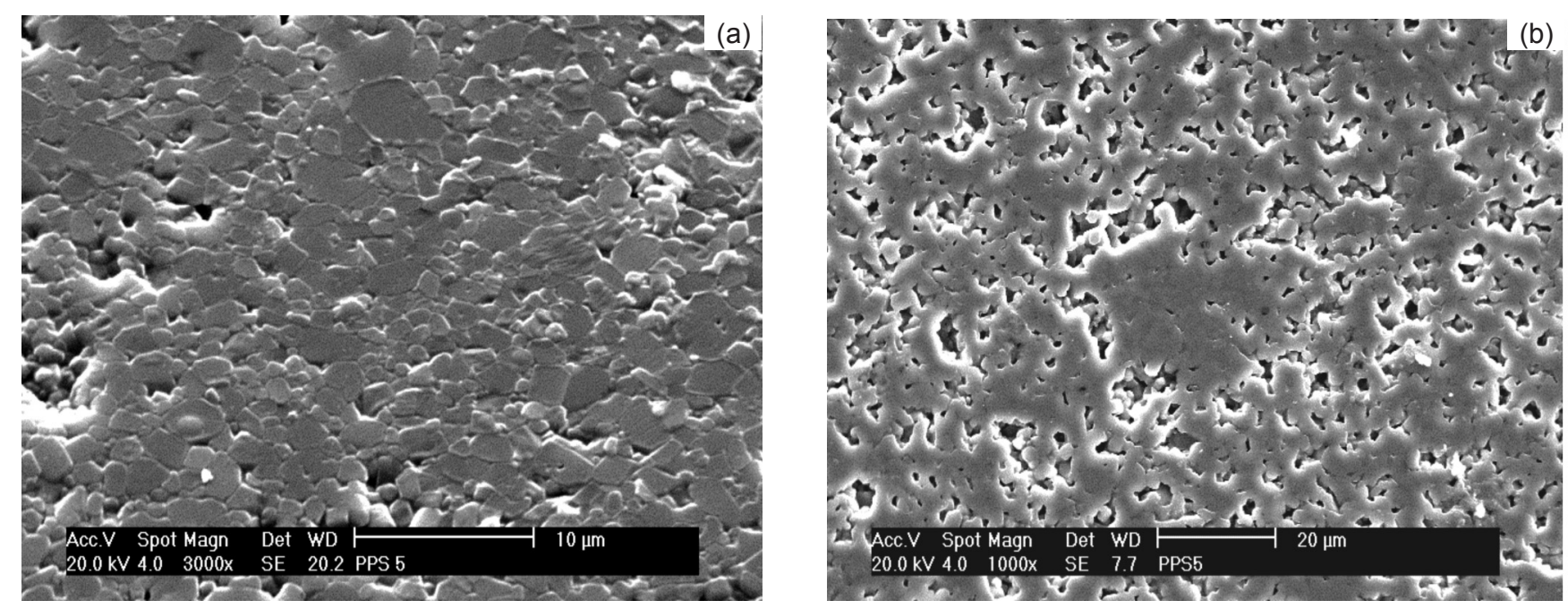

Figura 8: Micrografias obtidas por MEV da amostra A5: (a), (b)- superfícies polidas e atacadas.

[Figure 8: SEM micrographs of A5 samples: (a), (b)- surfaces polished and etched.] 
com polímero precursor. Isto acontece devido ao fato da densidade da alumina ser superior à densidade do $\mathrm{Si}_{2} \mathrm{Al}_{4} \mathrm{O}_{4} \mathrm{~N}_{4}$ e foi tomada apenas a densidade do $\mathrm{Al}_{2} \mathrm{O}_{3}$ como referência, já que não foi possível quantificar as outras fases cristalinas. Em trabalho comparando nanocompósitos de $\mathrm{Al}_{2} \mathrm{O}_{3}-\mathrm{SiC}$ obtidos por polímero precursor e pelo método convencional, sendo $1700{ }^{\circ} \mathrm{C}$ a temperatura de sinterização, a densidade do material preparado com polímero foi ligeiramente inferior à do nanocompósito obtido pelo método tradicional [35], dados estes que estão de acordo com os resultados deste trabalho. As perdas de massa das amostras $\mathrm{Al}_{2} \mathrm{O}_{3}$ :PPS foram relativamente baixas.

A quantidade de carbono remanescente para todas as amostras foi inferior a $0,8 \%$, o que é um aspecto positivo já que o mesmo pode produzir gás $\mathrm{CO}$, formado pela reação entre $\mathrm{SiO}_{2}$ e C, podendo ficar aprisionado nos poros
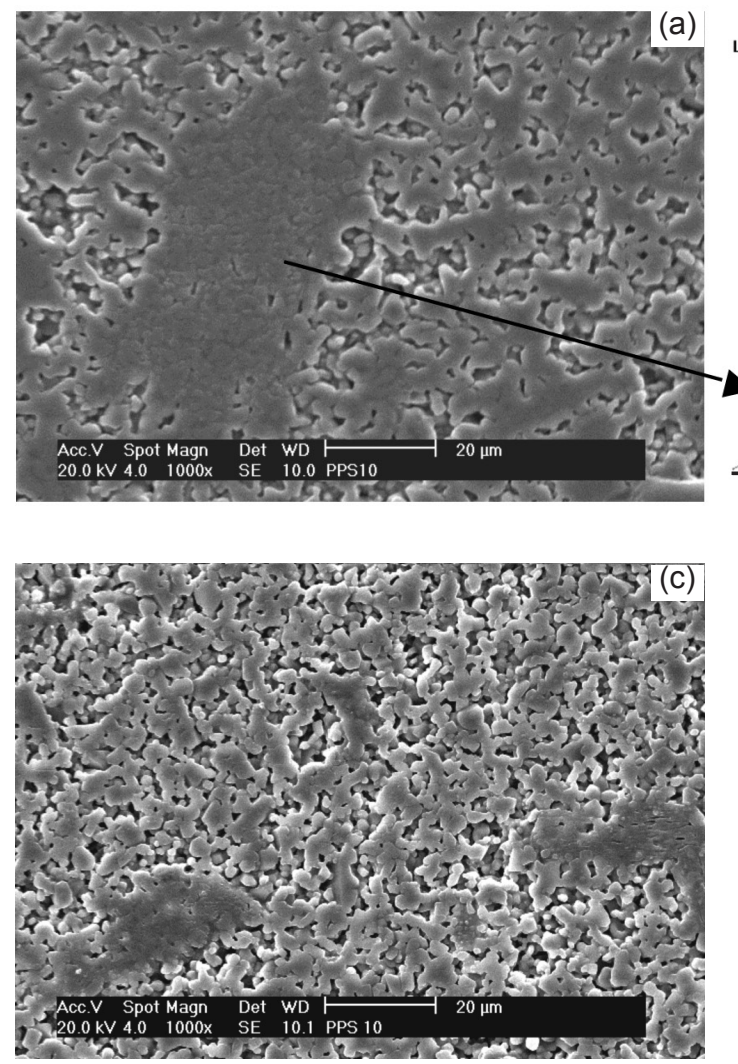

Untitled:2 Label A:

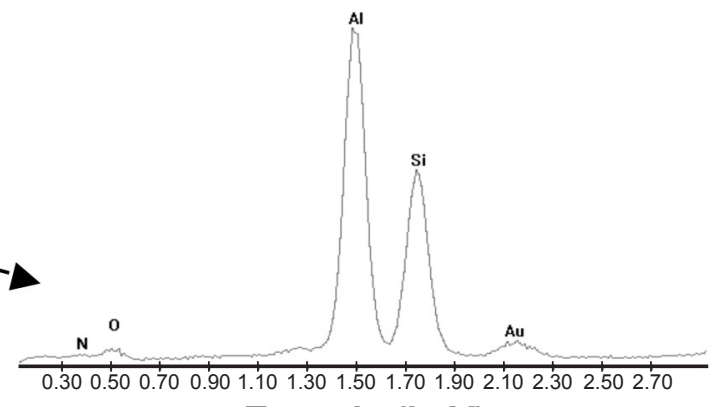

(b)

\section{Energia (keV)}
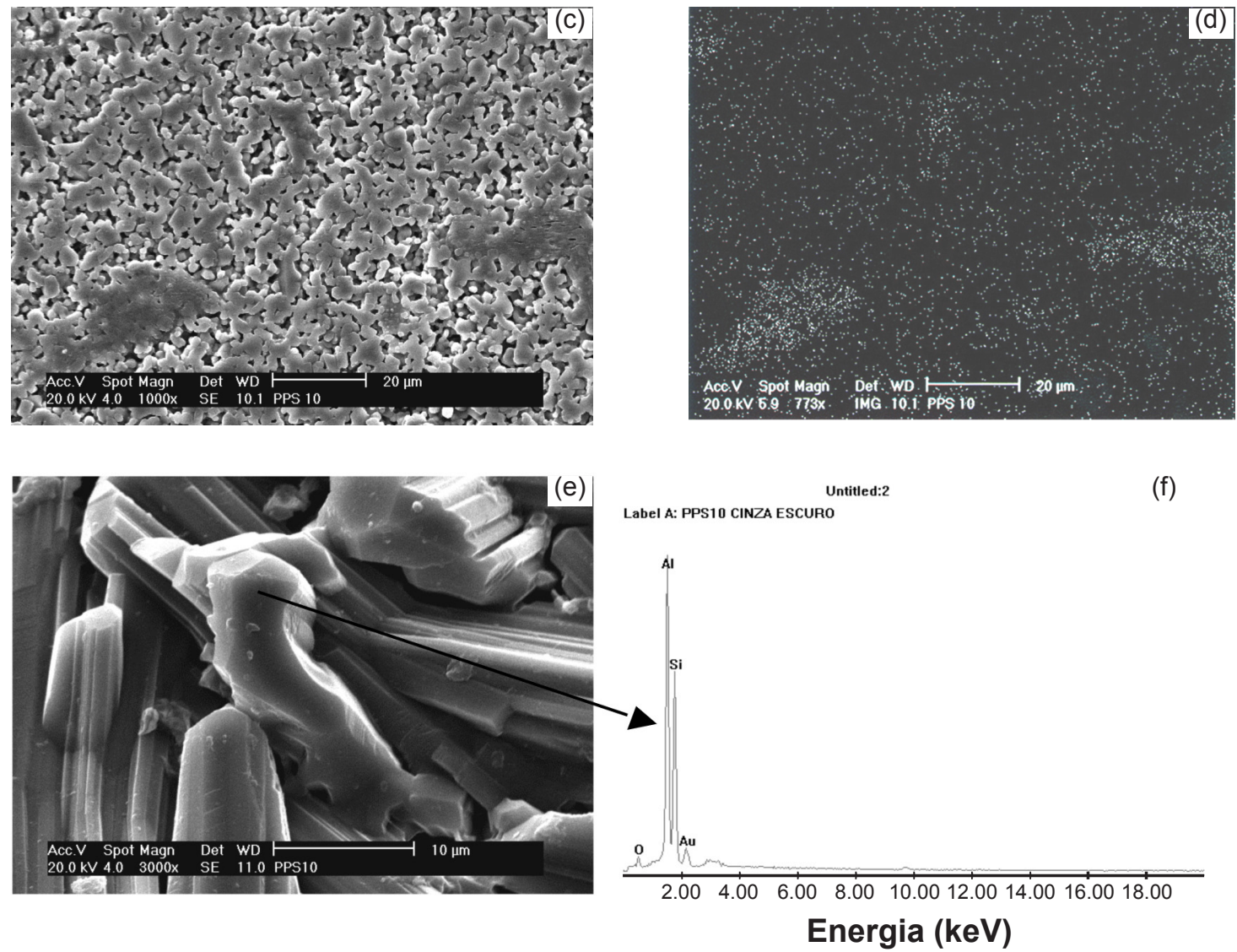

Figura 9: Micrografias obtidas por MEV da amostra A10: (a) amostra polida e atacada; (b) espectro de EDS da região sem poros, item (a); (c) amostra polida e atacada; (d) mapeamento de raios X de Si da região apresentada no item (c); (e) superfície de fratura; (f) espectro de EDS da amostra apresentada no item (e).

[Figure 9: SEM micrographs of A10 sample: (a) polished and etched specimen; (b) EDS spectrum of region without pores, item (a); (c) polished and etched specimen; (d) Si X-ray mapping of region presented in item (c); (e) surface fracture; (f) EDS spectrum of region presented in item (e).] 
Tabela II - Valores de dureza e de tenacidade à fratura das amostras A0, A1, A5 e A10.

[Table II - Vickers hardness and thoughness of A0, A1, A5 and AlO specimens.]

\begin{tabular}{ccc}
\hline Amostra & $\begin{array}{c}\mathrm{H}_{\mathrm{v}} \\
(\mathrm{GPa})\end{array}$ & $\begin{array}{c}\mathrm{K}_{\mathrm{Ic}} \\
\left(\mathrm{MPa} \cdot \mathrm{m}^{1 / 2}\right)\end{array}$ \\
\hline A0 & $14,9 \pm 0,6$ & $3,95 \pm 0,13$ \\
A1 & $15,4 \pm 0,8$ & $3,81 \pm 0,15$ \\
A5 & $15,2 \pm 0,6$ & $4,11 \pm 0.13$ \\
A10 & $12,8 \pm 1,3$ & $3,99 \pm 0,18$ \\
\hline
\end{tabular}

fechados, o que prejudicaria a sinterização destes materiais [36]. O carbono livre pode também inibir a sinterização reagindo com a matriz de $\mathrm{Al}_{2} \mathrm{O}_{3}$ a altas temperaturas [36]. $\mathrm{O}$ comportamento e o papel desempenhado pelo carbono livre que aparece durante a pirólise de polissiloxanos têm sido pouco estudados e as discussões são muito controversas [37, 38]. Na Fig. 6 é mostrada a variação no teor de carbono com a quantidade de polímero das amostras A1, A5 e A10, após sinterização.

À medida que a quantidade de polímero aumenta, o teor de carbono também aumenta, atingindo cerca de $0,7 \%$ em massa para a amostra A10.

Na Fig. 7 são mostradas as micrografias obtidas por MEV da amostra A1 e o resultado da análise por EDS para algumas regiões selecionadas (indicadas por setas). O espectro de EDS da Fig. 7b é relativo à região cinza escuro marcada em (a), apresentando picos de $\mathrm{Si}, \mathrm{O}, \mathrm{Al}$, que sugerem a formação da fase $\mathrm{Si}_{2} \mathrm{Al}_{4} \mathrm{O}_{4} \mathrm{~N}_{4}$, a fase secundária está distribuída de maneira relativamente homogênea na matriz. $\mathrm{O}$ espectro relativo à matriz, Fig. 7d, possui apenas os picos devido a $\mathrm{Al}$ e $\mathrm{O}$, indicando $\mathrm{Al}_{2} \mathrm{O}_{3}$. Alguns poros podem ser observados.

Na Fig. 8 são mostradas as micrografias, obtidas por MEV, da amostra A5. As Figs. 8a e 8b mostram superfícies polidas, atacadas a vácuo, a $1550{ }^{\circ} \mathrm{C}$ por $15 \mathrm{~min}$, nas quais se observam poros e prováveis arrancamentos provocados durante o polimento.

Na Fig. 9 são mostradas micrografias obtidas por MEV, EDS e mapeamento de raios X da amostra A10. Na Fig. 9 b é mostrado o espectro de EDS da região mais densa apresentada na micrografia (a); podem ser observados os picos bem definidos de $\mathrm{Si}, \mathrm{Al}$ e O, provavelmente relativos à fase formada $\mathrm{Si}_{2} \mathrm{Al}_{4} \mathrm{O}_{4} \mathrm{~N}_{4}$, de acordo com resultados de difração de raios X, Fig. 5. Na Fig. 9d pode-se observar mapeamento de raios $\mathrm{X}$ do $\mathrm{Si}$, referente à região da amostra apresentada em (c). Nota-se que no mapeamento de raios $\mathrm{X}$ existem áreas ricas em $\mathrm{Si}$, que coincidem com as áreas mais densas da micrografia. Conclui-se, portanto, que a fase à base de Si não está distribuída uniformemente na matriz, mas sim na forma de aglomerados. Na Fig. If é mostrado o espectro de EDS da região da micrografia (e), podendo-se observar o pico de Si.

As amostras, com exceção da amostra $\mathrm{A} 1$, não são homogêneas, como a série de micrografias apresentadas evidencia. De acordo com a literatura a composição química do produto final e a microestrutura são fortemente influenciadas pela quantidade de polímero empregada, pelos grupos funcionais dos polímeros precursores, pelas condições da pirólise [23, 38-40] e da sinterização, ou seja, basicamente pela temperatura, atmosfera e tempo de tratamento térmico. Uma característica comum a todas as amostras é a presença de poros devida, principalmente, à volatilização dos produtos orgânicos durante a pirólise [41].

$\mathrm{Na}$ Tabela II são apresentados os valores de dureza Vickers e tenacidade à fratura das amostras A0, A1, A5 e A10. A dureza da amostra padrão foi inferior às durezas das amostras $\mathrm{A} 1$ e $\mathrm{A} 5$, porém superior à dureza da A10. Em relação à tenacidade a fratura, a amostra $\mathrm{A} 5$ apresentou um valor maior que as outras amostras, inclusive em relação à amostra padrão.

Os valores de dureza variaram na faixa de $12,8 \mathrm{GPa}$ a $15,4 \mathrm{GPa}$. De acordo com a literatura os valores de dureza do $\mathrm{Al}_{2} \mathrm{O}_{3}$ variam muito, podendo atingir $23 \mathrm{GPa}$, dependendo do processamento e do método de medida. A dureza depende essencialmente da porosidade e do tamanho de grão do material. Esta propriedade é influenciada, também, por defeitos e micro-trincas que possam estar sob a indentação [42].

Uma das vantagens de preparar materiais por este método é que a conformação é relativamente simples, já que no processo de obtenção das amostras é formada uma mistura levemente pastosa, que possibilita verter o material em matrizes de diferentes formas.

\section{CONCLUSÕES}

As amostras contêm, além de $\mathrm{Al}_{2} \mathrm{O}_{3}, \mathrm{Si}_{2} \mathrm{Al}_{4} \mathrm{O}_{4} \mathrm{~N}_{4}$, que é uma fase formada com os produtos de decomposição do polímero, como $\mathrm{Si}, \mathrm{O}$ e com a atmosfera de $\mathrm{N}_{2}$. A fase encontrada é relevante para o trabalho, pois possui alta resistência à flexão e ao desgaste. A obtenção de compósitos cerâmicos utilizando polímeros precursores mostrou-se viável para materiais à base de alumina, com grande potencial para a obtenção de peças com geometria complexa.

\section{AGRADECIMENTOS}

Ao CNPq pelo apoio financeiro, ao CCN/IPEN pelos ensaios de picnometria de hélio e ao Departamento de Engenharia Mecânica da Escola Politécnica da USP por disponibilizar os equipamentos para os ensaios de dureza.

\section{REFERÊNCIAS}

[1] A. Okada, J. Eur. Ceram. Soc. 28 (2008) 1097.

[2] W. Acchar, D. M. B. Wolff, Mat. Sci. Eng. A 396 (2005) 251.

[3] M. A. Diniz, W. Acchar, Y. A. A. Fonseca, C. R. C. Sousa, E. G. Ramalho, Anais do $52^{\circ}$ Congresso Brasileiro de Cerâmica, Florianópolis, SC (2008).

[4] H. Schmidt, P. Buhler, P. Greil, In: Proc. Conf. ECRS, 
Riccione, Itália (1995).

[5] P. Greil, J. Am. Ceram. Soc. 78 (1995) 835.

[6] S. Dire, R. Ceccato, F. Babonneau, J. Sol-Gel Sci. Tech. 34, 1 (2005) 53.

[7] W. Acchar, M. A. Diniz, Y. A. A. Fonseca, C. R. C. Sousa, E. S. Lima, J. Mater. Sci. 43 (2008) 597.

[8] J. S. Lee, T. Yano, J. Eur. Ceram. Soc. 24 (2004) 25.

[9] P. Colombo, T. E. Paulson, C. G. Pantano, J. Am. Ceram. Soc. 80 (1997) 2333.

[10] P. Colombo, T. Gambaryan-Roisaman, M. Scheffler, P. Buhler, P. Greil, J. Am. Ceram. Soc. 84, 10 (2001) 2265.

[11] M. Ade, J. Haubelt, J. Eur. Ceram. Soc. 23 (2003) 1979.

[12] W. Qiao, M. S. Y. Lim, S. H. Yoon, I. Mochida, L. C. Ling, J. H. Yang, Appl. Surf. Sci. 253 (2007) 4467.

[13] O. Goerke, E. Feike, T. Heine, A. Trampert, H. Schubert, J. Eur. Ceram. Soc. 24 (2004) 2141.

[14] M. A. Schiavon, K. J. Cuiffi, I. V. P. Yoshida, J. NonCryst. Sol. 353 (2007) 156.

[15] J. Bill, F. Aldinger, Precursor - derived covalent ceramics: Synthesis, Structures and High Temperature Mechanical Properties, Eds. J. Bill, F. Wakai, F. Aldinger, Wiley-VCH, Weinheim, Alemanha (1999) 35.

[16] A. Rahimi, P. Shokolahi, Int. J. Inorg. Mat. 3 (2001) 843.

[17] B. Hardman, A. Torkelson, Silicones, in Encyclopedia of polymer science and engineering, Ed. J. I. Kroschvitz, John Wiley \& Sons, New York, EUA, 15 (1989) 205.

[18] A. Rahimi, P. Shokolahi, Int. J. Inorg. Mat. 3 (2001) 843.

[19] M. A. Schiavon, S. U. A. Redondo, S. R. O. Pina, I. V. P. Yoshida, J. Non-Cryst. Sol. 304 (2002) 92.

[20] C. Czosnek, J. Wolszezak, M. Drygás Góra, J. F. Janik, J. Phys. Chem. Sol. 65 (2004) 647.

[21] T. Erny, D. Suttor, P. Greil, Properties of novel polymer derived ceramic composites, Proc. $4^{\text {th }}$ Eur. Ceram. Soc. Conf., Itália (1995).

[22] C. Liu, J. Zhang, J. Sun, X. Zhang, Y. Hu, Ceram. Int. 33, 7 (2007) 1149.

[23] H. Björklund, Grain Morphology and Intergranular Structure of $\mathrm{Si}_{3} \mathrm{~N}_{4}$ and $\mathrm{Al}_{2} \mathrm{O}_{3}$ Based Ceramics, $\mathrm{PhD}$ Thesis, Chalmers University of Technology, Inglaterra (1996).

[24] L. D. Hart, Alumina chemicals; science and technology handbook, in Production, processes, properties, and applications for calcined and high-purity alumina, Ed. T. J. Carbone, The Am. Ceram. Soc., EUA (1990) 99.

[25] L. M. Epure, S. Dimitrievska, Y. Merhi, J. Biom. Mater. Res., Part A, 83, 4 (2007) 1009.

[26] R. M. Rocha, P. Greil, J. C. Bressiani, A. H. A. Bressiani, Mater. Res. 8, 2 (2005) 191.

[27] C. B. Ponton, R. D. Rawlings, Mater. Sci. Tech. 5 (1989) 865 .

[28] G. R. Anstis, P. Chantikul, B. R. Lawn, D. B. Marshall, J. Am. Ceram. Soc. 64, 9 (1981) 533.

[29] A. L. Smith, Analysis of Silicones, Ed. Wiley, EUA (1974).

[30] M. A. Schiavon, Polissiloxanos e polissilazanos como precursors de materiais cerâmicos e suas aplicações na obtenção de compósitos de matriz cerâmica, Tese Dr., Instituto de Química, UNICAMP, Campinas, SP (2002).

[31] J. Cordelair, P. Greil, J. Eur. Ceram. Soc. 20 (2000) 1947.

[32] R. M. Rocha, Obtenção e caracterização de cerâmicas no sistema Si-Al-O-N-C empregando pirólise de misturas de polissiloxano e cargas, Tese Dr., Instituto de Pesquisas Energéticas e Nucleares, S. Paulo, SP (2004).

[33] J. D. Jovanovic, M. N. Govedarica, P. R. Dvornic, I. G. Popovic, Pol. Degr. Stab. 61 (1998) 87.

[34] K. J. Negita, Am. Ceram. Soc. 69 (1986) c308-310.

[35] C. E. Borsa, R. J. Brook, Fabrication of $\mathrm{Al}_{2} \mathrm{O}_{3} / \mathrm{SiC}$ nanocomposites using a polymeric precursor, Ceramic processing science and technology, Ceramic Trans. 51, Eds. H. Hausner, G. L. Messing, S. Hirano, The Am. Ceram. Soc., EUA (1995) 653.

[36] J. S. Ha, C. S. Lim, C. S. Kim, D. S. Cheong, Met. Chem. Phys. 75 (2002) 241.

[37] R. Harshe, C. Balan, R. Riedel, J. Eur. Ceram. Soc. 24 (2004) 3471.

[38] C. G. Pantano, A. K. Sing, H. Zhang, J. Sol-Gel Sci. Technol. 14 (1999) 25.

[39] S. Trassl, G. Motz, E. Rössler, G. Ziegler, J. Am. Ceram. Soc. 85, 1 (2002) 239.

[40] K. J. Wynne, R. W. Rice, Ann. Rev. Mater. Sci. 14 (1984) 297.

[41] [27] M. Hörz, A. Zern, F. Berger, J. Haug, K. Müller, F. Aldinger, M. Weinman, J. Eur. Ceram. Soc. 25 (2005) 99.

[42] R. Warren, Ceramic-Matrix Composites, Ed. Chapman and Hall, EUA (1992).

(Rec. 26/03/2009, Ac. 16/07/2009) 